de la simplicité évangélique. Dans l'ensemble du texte se crée ainsi une «Vie de Jésus-Christ selon L'Arétin » où la voix du narrateur occupe une place centrale qui à la fois explique, édifie et cherche à émouvoir ; Jésus est présenté comme fondateur de la doctrine catholique, principalement de la doctrine catholique du salut. Mais il est aussi un homme beau, un homme doux souvent cerné par des foules, placé au cœur d'une nature qui participe pleinement à l'action.

La conclusion souligne la diversité des modalités dans la réécriture arétinienne des textes saints, diversité qui va de la liberté d'une harmonisation des sources retenues à une paraphrase épousant très étroitement le texte biblique. Bibliographie et index complètent les pages d'études.

Ce parcours très fin et minutieux des textes, effectué au gré de citations abondantes - on regrettera que certaines ne soient proposées qu'en traduction —, s'il n'évite pas longueurs ou répétitions, permet de mesurer le travail d'écriture d'un homme de Lettres autant que l'exacte portée de textes dont le succès fut grand, et bien au delà des frontières italiennes. Il permet aussi de reconsidérer l'image de L'Arétin, trop souvent réduite à son œuvre profane, et sans doute par ce biais de reconsidérer la place particulière de la littérature de spiritualité dans la période qui précède immédiatement l'ouverture du Concile.

BRUNO PETEY-GIRARD, Université de Paris 12 - Val de Marne

\title{
Jacques-Auguste de Thou
}

\section{La vie de Jacques-Auguste de Thou. I Aug. Thuani vita}

Introduction, établissement du texte, traduction et notes par Anne TeissierEnsminger

Paris : Honoré Champion, Textes de la Renaissance 126, 2007, 1085 p.

$\ll(\mathrm{R})$ établir le texte de la Vita dans toutes les sinuosités de son histoire paléographique $\gg(121)$, telle est l'ambition d'A. Teissier-Ensminger à la source de cette volumineuse édition critique. Un simple aperçu de l'opus permet d'apprécier à sa juste valeur l'importance de la tâche entreprise et le soin apporté à son élaboration : l'introduction couvre près de 175 pages (9-184) ; un résumé analytique de l'argument de chaque livre permet au lecteur d'embrasser aisément la matière (1009-1028); à quoi s'ajoutent un synopsis métrique (1031-1033), un récapitulatif des itinéraires 
(1035-1040), un index des noms de personnes (1041-1064) et un index des noms de lieux $(1065-1081)$.

Un tel travail s'avérait nécessaire ; en effet, aucune édition de la Vita n'avait vu le jour depuis plus de deux siècles. Par ailleurs, cette œuvre maintes fois sollicitée s'offrait jusqu'ici au lecteur curieux et au chercheur dans un état textuel peu acceptable, et, pour sa traduction en français, à travers le prisme déformant des « belles infidèles ». L'éditrice a donc repris l'ensemble des leçons manuscrites et s'est attachée à rendre scrupuleusement compte des remaniements opérés dans l'editio princeps de 1621. La traduction est assortie d'un appareil critique corrigeant approximations et confusions affectant aussi bien la relation des événements que les références à certains personnages. Enfin, A. Teissier-Ensminger s'est attachée à mettre en évidence les aspects saillants de la personnalité de l'auteur, telle que peut les révéler une étude attentive de la Vita ; apparaît alors un Jacques-Auguste de Thou juriste, historien et poète, dont l'œuvre au destin paradoxal offre un « échantillon significatif de jurisécriture » (15) qu'il convient de prendre en compte désormais afin d'en mieux apprécier la portée.

Première étape de l'itinéraire auquel nous convie la riche introduction : l'étude de la genèse et de l'élaboration des éditions. L'incertitude concernant la datation du texte autographe et le livre VI est en grande partie résolue ; pour le premier, le terminus a quo est fixé à la $2^{\text {nde }}$ moitié de 1614 ; pour le dernier livre, à la $1{ }^{\text {ère }}$ moitié de 1615 (21). Sont abordés ensuite les problèmes liés à la présence de deux titres : le titre long concerne le premier livre et donne des indications sur son contenu et sa dimension juridique ; le titre court embrasse l'ensemble de l'œuvre. L'éditrice s'attarde également sur la composition complexe d'un texte marqué par plusieurs événements : l'éviction de J.-A de Thou de la charge de premier président du Parlement de Paris, la condamnation de l'Histoire, l'assassinat d'Henri IV et le décès de l'épouse de l'auteur. Soulignant le nombre élevé de références à l'Histoire, la critique démontre d'une manière pertinente la fonction de justification de la Vita, « histoire d'une vie, mais surtout vie de l'Histoire $\gg$, selon une formule heureuse (35).

L'examen des manuscrits retient ensuite toute l'attention d'A. TeissierEnsminger, qui privilégie, après la précieuse contribution de $S$. Kinser, les traits paléographiques, et analyse minutieusement quatre manuscrits : le manuscrit autographe $(\ll \mathrm{A} \gg)$; la copie « simple $»$, relativement hétérogène $(\ll \mathrm{M} \gg)$; la copie révisée $(\ll \mathrm{R} \gg)$ et le manuscrit français $(\ll \mathrm{T} \gg)$, qu'il est possible d'attribuer à Pierre Dupuy. La question concernant l'établissement du texte proprement dit, soulève, quant à elle, autant de problèmes - chronologie incertaine, confiance relative à accorder à la copie révisée, « trou $\gg$ entre l'editio princeps et l'état du texte de $\ll \mathrm{R} \gg$ 
après révision — qu'elle présente de points forts, à savoir : le fait de disposer de trois états du texte, d'avoir des copies indépendantes et de bénéficier ainsi d'une grande complémentarité des états du texte.

En dernier lieu, l'éditrice se propose d'étudier de plus près les caractéristiques juridique, historique et autobiographique de la Vita, en insistant d'abord sur la dimension « jurislittéraire » (125), occultée jusqu'ici au profit de la seule prise en considération du caractère autobiographique. Un deuxième modèle apparaît, celui des historiens : César, Plutarque, mais aussi Suétone et Tacite. Les Vies de saints constituent un troisième modèle, attestant ainsi la richesse d'un texte convoquant toute une palette de savoirs historiques, théologiques littéraires, médicaux et juridiques en vue d'éclaircir et de défendre la somme qu'est l'Histoire.

Au terme d'un parcours inaugural livrant au lecteur toutes les informations nécessaires à la bonne compréhension de la Vita, sans qu'à aucun moment les questions en suspens ne soient occultées, le lecteur peut goûter enfin un texte jusqu'ici difficilement accessible, et ce dans une édition qui satisfait désormais pleinement aux exigences universitaires. La mise en page du texte est claire, et la traduction en miroir, précise et élégante, comme l'atteste, par exemple, celle du poème que J.-A. de Thou adresse « Au très illustre Prince Charles, cardinal de Vendôme » (576-585). Si l'on peut regretter l'absence d'une bibliographie, sinon systématique, du moins susceptible de rendre service à l'amateur éclairé comme au jeune chercheur perdu dans le dédale des références, force est d'admettre que l'établissement d'un appareil de notes précises et copieuses ainsi que les renvois à des ouvrages offrant une bibliographie thuanienne importante et récente (14) viennent finalement combler nos attentes. Nul doute, cette édition de la Vita fait date.

Thierry victoria, Classes Préparatoires aux Grandes Écoles, Poitiers ; CEMar, Université de Picardie-Jules Verne.

\section{Patronnes et mécènes en France à la Renaissance}

Études réunies et présentées par Kathleen Wilson-Chevalier avec la collaboration d'Eugénie Pascal.

St-Étienne : Publications de l'Université de Saint-Etienne, L'École du genre, 2007, $682 \mathrm{p}$.

Vingt-cinq seiziémistes ont contribué à ce volume qui met en lumière les femmes, auteurs ou non, commanditaires ou protectrices d'auteurs et d'artistes sinon 